\title{
Search Performance Prediction for Multistatic Sensor Fields
}

\author{
Michael J. Walsh \\ Naval Undersea Warfare Center \\ Newport, Rhode Island \\ m.j.walsh@ieee.org
}

\author{
Thomas A. Wettergren \\ Naval Undersea Warfare Center \\ Newport, Rhode Island \\ t.a.wettergren@ieee.org
}

\begin{abstract}
An existing search performance model for distributed passive sensor networks is extended to multistatic sensor networks comprised of active sources and passive receivers. The model provides an upper bound on the detection range of a receiver in the field, and uses this bound to compute the expected probability of successful search for a given target track. This expected probability depends on the numbers of sources and receivers in the field, their location distribution functions, and the location and orientation of the track. For uniformly (randomly) distributed sources and receivers, the distribution function for the upper bound on receiver detection range can be computed analytically in closed form. We show that the resulting estimates of probability of successful search for uniformly distributed multistatic fields is a reasonable approximation to more accurate values obtained via Monte Carlo simulation for various numbers of sources and receivers, and for various numbers of required detections. We conclude with a discussion of how this model may be used in designing and planning multistatic systems for undersea surveillance.
\end{abstract}

\section{INTRODUCTION}

Automated detection of submerged moving targets is a fundamental problem in undersea surveillance. Distributed sets of unattended sensors provide a tremendous opportunity to fulfill this mission in a cost-effective manner [1]. However, to fully realize the benefits of spatially distributed sensors in the undersea domain, the potential gains of a multistatic system, comprised of a mixture of active sources and passive receivers must be considered. It has been conjectured that such systems may provide the best engineering design trade-offs, especially for large search areas where the system design is constrained by total cost. Development of useful tools to facilitate design analysis studies is critical to realizing the potential benefit of multistatic systems to ocean monitoring.

An enduring problem in the analysis of multistatic systems for undersea surveillance has been the lack of (even approximate) closed-form analytical models of detection performance. For design trade-off studies, such models are preferable to simulation-based approaches (such as Monte Carlo approaches), as they provide a mechanism to examine complex parametric inter-dependencies in a manner that is computationally unfeasible for strictly simulation-based studies of these complex systems.

Our model for the detection performance of a multistatic sensor field (or, more accurately, the search effectiveness of the field, with successful search defined as a sequence of target detections) is an extension of the model developed in [2] for passive sensor fields. It is also similar in spirit to, and shares common elements with, the Multistatic Performance Prediction Methodology (MPPM) of Bowen and Mitnick [3]. Their performance model focuses on the probability that a square grid of active sources and uniformly dispersed receivers detects a target confined to a grid cell for a fixed number of ping cycles. Their model, like ours, uses a bistatic version of the sonar equation to determine areas of the surveillance region that permit target detection by a receiver in the field. Both models also use similar probabilistic arguments to construct measures of field performance based on source and receiver densities, though the two approaches differ significantly in their notion of density.

Sensor density in [3] is taken to mean the number of sources or receivers per unit area for uniformly (deterministically) distributed sensors. Sources and receivers in our model are assumed to be distributed according to spatial probability density functions, so that their locations are not known precisely. Also, the target in our approach is not confined to a sector of the surveillance region, and our measure of system performance (search effectiveness) is an explicit function of the number of required detections. Single detection search effectiveness of a fixed grid of regularly spaced sources and uniformly (deterministically) dispersed receivers as in [3] is a special case of our model.

The multistatic search effectiveness model that we develop is an approximation that provides an upper bound on multistatic system performance in terms of engineering design parameters. Thus, the model can be used by designers both to select important system characteristics (i.e., source strength, receiver gains, etc.), and to plan the specific deployment of a multistatic field (i.e., source and receiver locations), as described in [4]. In the next section, we review the analytical framework developed in [2] for assessing the performance of a distributed passive sensor network searching for a moving target. Then, in Section III, the methodology is extended to the multistatic problem through a sequence of approximations. In Section IV we provide example calculations for uniformly (randomly) distributed sources and receivers, and compare these results to detailed (yet computationally cumbersome) Monte Carlo simulations. We conclude in Section V with recommendations for future work. 


\section{REVIEW OF APPROACH FOR PASSIVE SENSOR FiELDS}

\section{A. Receiver Detection Range}

Consider a field of $N_{R}$ receivers deployed in a region $\mathcal{A} \subset \mathbb{R}^{2}$ to search for a target moving with constant speed $V$ and constant course $\theta$ over a time interval $\mathcal{T}=\left\{t: t_{0} \leq\right.$ $\left.t \leq t_{0}+\Delta t\right\}$. The target location at any time $t \in \mathcal{T}$ is given by $\vec{x}_{T}(t) \in \mathcal{A}$. It is assumed that the receiver locations are fixed, and that each receiver has the same search capability, characterized by its ability to detect the target as it moves in proximity to the receiver location. In particular, it is assumed that the $j$ th receiver, at location $\vec{x}_{R_{j}}$, detects the target with probability $P_{d}$ if the target comes within the detection range $R_{d}$ of the receiver sometime during the search interval $\mathcal{T}$. Using results from [5, Chapters 2 and 5], the detection range $R_{d}$ of a receiver can be determined from the passive sonar equation. Specifically, if the target has a source level, in decibels, of at least $S L$, then the $j$ th receiver detects the target at time $t$ with probability $P_{d}$ if

$$
S L-T L\left(\vec{x}_{T}(t), \vec{x}_{R_{j}}\right) \geq T H,
$$

where $T L$ is the transmission loss between points $\vec{x}_{T}(t)$ and $\vec{x}_{R_{j}}$, and $T H$ is an energy threshold that depends on the environmental characteristics of the search region $\mathcal{A}$ (such as ambient noise level), the physical characteristics of the receiver (such as array gain), and detection and false alarm requirements. If we assume further that the environment is nearly uniform, such that the transmission loss between two points in $\mathcal{A}$ follows a simple power law, then the $j$ th receiver detects the target at time $t$ with probability $P_{d}$ if

$$
S L-m \log r_{R_{j}}(t) \geq T H
$$

for some constant $m>0$, where $r_{R_{j}}(t)$ is the distance from the target to the $j$ th receiver at time $t$. Solving this equation for $r_{R_{j}}(t)$ gives

$$
r_{R_{j}}(t) \leq 10^{\frac{1}{m}(S L-T H)} \equiv R_{d} .
$$

For a passive sensor field and for the simplifying assumptions listed above, the receiver detection range $R_{d}$ is a constant. In Section III, we show that the receiver detection range for a multistatic sensor field is not a constant, but depends on the location of the target, the number of sources, and the source locations.

\section{B. Receiver Detection Region for a Moving Target}

For the purpose of this paper, it is convenient to express detection events, and regions of $\mathcal{A}$ where receivers can detect the target, in terms of set membership and set unions, respectively. Let $\Omega_{t} \subset \mathcal{A}$ denote the set of all possible receiver locations that permit target detection at time $t$, that is, the set of all points in $\mathcal{A}$ that satisfy the inequality (3):

$$
\Omega_{t}=\left\{\vec{x} \in \mathcal{A}:\left\|\vec{x}-\vec{x}_{T}(t)\right\| \leq R_{d}\right\} .
$$

Then the $j$ th receiver detects the target with probability $P_{d}$ at time $t$ if $\vec{x}_{R_{j}} \in \Omega_{t}$. It follows that the $j$ th receiver detects

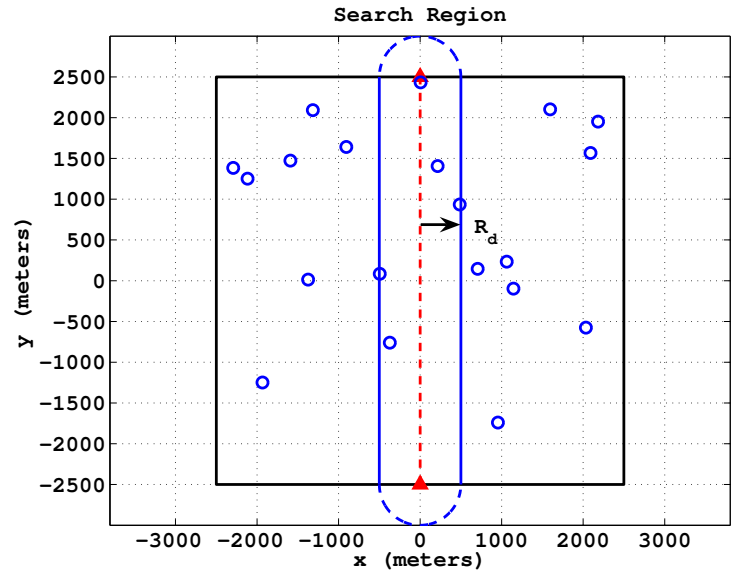

Fig. 1. Detection region $\Omega$ (solid blue lines) about target track for a passive sensor field of receivers, each with detection range $R_{d}$.

the target with probability $P_{d}$ at least once during the search interval if

$$
\vec{x}_{R_{j}} \in \bigcup_{t \in \mathcal{T}} \Omega_{t} \equiv \Omega .
$$

The detection region $\Omega \subset \mathcal{A}$ is depicted in Figure 1 for a target moving along the $y$-axis through the middle of a square search region $\mathcal{A}$. We show in Section III that for a multistatic system, the shape of the detection region $\Omega$ is, in general, much more complicated than the "pill" shaped region of Figure 1, and in fact depends on the distribution of sources in the search region.

\section{Probability of Successful Search for Receiver Network}

In this paper, as in [2], the measure of search effectiveness of the sensor field is the probability of successful search, denoted $P_{S S}(k)$, and defined as the probability of getting at least $k$ detections (from $k$ different receivers) during the search interval. In the special case where $P_{d}=1, P_{S S}(k)$ is the probability of finding at least $k$ of the $N_{R}$ receivers in the detection region $\Omega$. As described in [2], the field-level probability of successful search depends on the sensor-level probability of successful search, denoted $p$. This probability is given in [2] by

$$
p=1-\exp \left(-P_{d} \phi\right),
$$

where $\phi$ is the probability of finding a receiver in the detection region $\Omega$. If the receivers are randomly, independently and identically distributed in $\mathcal{A}$ according to the density function $g: \mathbb{R}^{2} \rightarrow \mathbb{R}^{+}$, then

$$
\phi=\int_{\Omega} g(\vec{x}) d \vec{x} .
$$

Thus, when the receivers are uniformly (randomly) distributed over the search region $\mathcal{A}$, the probability of finding a receiver in the detection region $\Omega$ is just the area of $\Omega$ divided by the area of $\mathcal{A}$. Given the sensor-level probability of successful search $p$, the field-level probability of successful search $P_{S S}(k)$ is defined in terms of $N_{R}$ independent Bernoulli trials 
with success probability $p$ and failure probability $1-p$. Thus, the probability of at least $k$ distinct receivers detecting during the search interval $\mathcal{T}$, is given by [6, Chapter 3]

$$
P_{S S}(k)=1-\sum_{\ell=0}^{k-1}\left(\begin{array}{c}
N_{R} \\
\ell
\end{array}\right) p^{\ell}(1-p)^{N_{R}-\ell}
$$

for $k=0,1, \ldots, N_{R}$, where $p$ is as in (6). From (6), (7), and (8), we see that the field-level probability of successful search $P_{S S}(k)$ for a passive system depends explicitly on a number of quantities, including the number of receivers $N_{R}$, the number of required detections $k$, and the detection region $\Omega$. We show in the next section that the determination of $P_{S S}(k)$ for a multistatic sensor field is considerably more complicated, as the receiver detection region $\Omega$ about the target track depends also on the number of sources, and their distribution over the search region.

\section{Extension of ApProach to Multistatic SENSOR FIELDS}

Assume, as in Section II, a field of $N_{R}$ fixed receivers, each with the same detection capability, independently and identically distributed over the region $\mathcal{A}$ according to the density function $g: \mathbb{R}^{2} \rightarrow \mathbb{R}^{+}$to search for a constant-velocity target over the time interval $\mathcal{T}$. In addition to the receiver field, consider a field of $N_{S}$ fixed sources, independently and identically distributed over $\mathcal{A}$ according to the different density function $f: \mathbb{R}^{2} \rightarrow \mathbb{R}^{+}$, each with the same source level $S L$ (in decibels). In this section, it is assumed that the search capability of a receiver is characterized by its ability to detect acoustic energy originating from a source and reflected off the target, and to discriminate this energy from that received directly from the source.

\section{A. Receiver Detection Range}

What is the detection range $R_{d}$ for a receiver in this multistatic sensor field? To answer this question, assume for the moment that the $i$ th source, at location $\vec{x}_{S_{i}}$, is the only source in the field active during the search interval $\mathcal{T}$, and that it produces a source level $S L\left(\vec{x}_{S_{i}}, \vec{x}_{T}(t)\right)$ at target location $\vec{x}_{T}(t)$. It is assumed that the distance traveled by the target in the time it takes sound to travel from the source to the target is negligible, and that the target reflects sound equally in all directions and without loss. (The latter assumptions are clearly unrealistic, but are adequate for the purpose of this paper, which is to provide an upper bound on the search effectiveness of a multistatic sensor field.) With these assumptions we have, from (1) through (3), that if the $i$ th source pings at time $t$ during the search interval $\mathcal{T}$, then the $j$ th receiver detects the target at $\vec{x}_{T}(t)$ with probability $P_{d}$ if

$$
r_{R_{j}}(t) \leq 10^{\frac{1}{m}\left[S L\left(\vec{x}_{S_{i}}, \vec{x}_{T}(t)\right)-T H\right]} \equiv R_{d}\left(\vec{x}_{S_{i}}, \vec{x}_{T}(t)\right) .
$$

Thus, for a bistatic sensor node, the receiver detection range $R_{d}$ is not a constant, but depends, in general, on the relative locations of the source and the target.

It will be convenient to rewrite (9) in terms of the range $r_{S_{i}}(t)$ from the $i$ th source to the target at time $t$, and the monostatic detection range $r_{0}$, the latter defined as the maximum range at which a monostatic sensor node (i.e., a colocated source/receiver pair) can detect the target. To proceed, we observe that the source level $S L\left(\vec{x}_{S_{i}}, \vec{x}_{T}(t)\right)$ at target location $\vec{x}_{T}(t)$ due to the $i$ th source at time $t$ is given by $S L-m \log r_{S_{i}}(t)$. Substituting this expression into (9) yields

$$
r_{S_{i}}(t) r_{R_{j}}(t) \leq 10^{\frac{1}{m}(S L-T H)} \equiv r_{0}^{2},
$$

a well-known inequality in both bistatic sonar and radar (see, for example, [7], [8]). For fixed and known source and receiver locations, the set of all target locations $\vec{x}_{T}(t)$ at time $t$ that satisfy the equality in (10) define a Cassini oval [9], the interior of which is the set of all target locations at time $t$ that are detectable by the source/receiver pair $\left(\vec{x}_{S_{i}}, \vec{x}_{R_{j}}\right)$.

If the $i$ th source is the only active source in the field during the search interval $\mathcal{T}$, then from (9) and (10) we have that the receiver detection range at time $t$ is a function of the range from the $i$ th source to the target at time $t$ :

$$
R_{d}\left(\vec{x}_{S_{i}}, \vec{x}_{T}(t)\right)=r_{0}^{2} /\left\|\vec{x}_{S_{i}}-\vec{x}_{T}(t)\right\|=r_{0}^{2} / r_{S_{i}}(t) .
$$

Suppose now that all $N_{S}$ sources are active during the search interval. What is their net effect on the receiver detection range? To answer this question, we will assume further that the distance traveled by the target in the time it takes to complete one ping cycle, during which each source pings exactly once, is small enough to assume that the sources ping simultaneously. Then, from (11), if the target at location $\vec{x}_{T}(t)$ is detected with probability $P_{d}$ at time $t$ by the source/receiver pair $\left(\vec{x}_{S_{i}}, \vec{x}_{R_{j}}\right)$, it is also detected with probability $P_{d}$ at time $t$ by the pair $\left(\vec{x}_{S_{(1)}}(t), \vec{x}_{R_{j}}\right)$, where $\vec{x}_{S_{(1)}}(t)$ denotes the location of the nearest source to the target at time $t$. Under this important simplifying assumption, the receiver detection range at time $t$ becomes

$$
R_{d}\left(\vec{x}_{S_{(1)}}(t), \vec{x}_{T}(t)\right)=r_{0}^{2} /\left\|\vec{x}_{S_{(1)}}(t)-\vec{x}_{T}(t)\right\|=r_{0}^{2} / r_{S_{(1)}}(t) .
$$

The assumption of a short ping cycle implies that either the number of sources in the field is relatively small, or that intelligent processing techniques are used to distinguish individual sources, as suggested in [10].

Since the source locations $\vec{x}_{S_{i}}$ are assumed to be independent and identically distributed random variables, it follows that the ranges $r_{S_{i}}(t)$ from the sources to the target at time $t$ are random variables as well. Let $f_{r(t)}: \mathbb{R}^{+} \rightarrow \mathbb{R}^{+}$denote the density function for the range from a source to the target at time $t$. This density function may be difficult to compute analytically even for the simplest source location distributions, since it depends on the shape of the search region $\mathcal{A}$ and the target location in the region. However, the density function $f_{r(t)}$ can always be approximated to any degree of accuracy by sampling from the source location density function $f$. Moreover, once an approximation to $f_{r(t)}$ has been obtained, the density function for the range $r_{S_{(1)}}(t)$ from the target to the nearest source at time $t$ can be obtained using the theory of order statistics. Indeed, the density function for the nearest 
range $r_{S_{(1)}}(t)$, given the density function $f_{r(t)}$ for the ranges $r_{S_{i}}(t)$, is given by [11, Theorem 5.5.2],

$$
f_{r_{(1)}(t)}(\rho)=N_{S} f_{r(t)}(\rho)\left[1-F_{r(t)}(\rho)\right]^{N_{S}-1}
$$

where $F_{r(t)}$ is the distribution function associated with the density function $f_{r(t)}$, that is,

$$
F_{r(t)}=\int_{0}^{\rho} f_{r(t)}(\tau) d \tau
$$

Given the density function (13) for the range from the target to the nearest source at time $t$, and expression (12) for the receiver detection range $R_{d}\left(\vec{x}_{S_{(1)}}(t), \vec{x}_{T}(t)\right)$ at time $t$ for the multistatic sensor field, we can, in principle, obtain the density function for $R_{d}$ (or at least an approximation to this function to any degree of accuracy by sampling from the source location density function $f$ ). Alternatively, the density function (13) can be used to compute moments of the receiver detection range $R_{d}$. In particular, the average value of $R_{d}\left(\vec{x}_{S_{(1)}}(t), \vec{x}_{T}(t)\right)$ at time $t$, denoted $\bar{R}_{d}\left(\vec{x}_{T}(t)\right)$, is given by

$$
\bar{R}_{d}\left(\vec{x}_{T}(t)\right)=r_{0}^{2} \int_{1}^{r_{0}^{2}} \frac{f_{r_{(1)}(t)}(\rho)}{\rho} d \rho .
$$

Hence, given the assumptions listed above, the average value of the receiver detection range $R_{d}$ is a function of the monostatic detection range $r_{0}$ and the location $\vec{x}_{T}(t)$ of the target in the search region $\mathcal{A}$; it depends implicitly on the number of sources $N_{S}$, and the source location density function $f$, through the density function $f_{r_{(1)}(t)}$ on range from the target to the nearest source.

\section{B. Receiver Detection Region for a Moving Target}

For multistatic sensor fields, the receiver detection region $\Omega$ about a moving target's track is complicated by the fact that the receiver detection range $R_{d}$ depends on the location of the track, the number of sources, and the distribution of the source locations in the field. Let $\Omega_{t} \subset \mathcal{A}$ denote the set of all possible receiver locations that permit target detection at time $t$, that is, the set of points in $\mathcal{A}$ that satisfy the inequality (9) for the nearest source to the target at time $t$ :

$$
\Omega_{t}=\left\{\vec{x} \in \mathcal{A}:\left\|\vec{x}-\vec{x}_{T}(t)\right\| \leq R_{d}\left(\vec{x}_{S_{(1)}}(t), \vec{x}_{T}(t)\right)\right\} .
$$

For a purely active system, the sources must ping in order for a receiver to detect the target. Let $\mathcal{S} \subset \mathcal{T}$ denote the ping schedule for the system, so that there is a detection opportunity at time $t$ if and only if $t \in \mathcal{S}$. Then the $j$ th receiver detects the target with probability $P_{d}$ at time $t \in \mathcal{S}$ if $\vec{x}_{R_{j}} \in \Omega_{t}$. Moreover, the $j$ th receiver detects the target with probability $P_{d}$ at least once during the search interval if

$$
\vec{x}_{R_{j}} \in \bigcup_{t \in \mathcal{S}} \Omega_{t} \equiv \Omega \text {. }
$$

The receiver detection region $\Omega$ is depicted in Figures 2 and 3 for the scenario of Figure 1 and for fixed and known source locations. The detection regions in these figures are for idealized multistatic fields with "continuous" ping schedules, that is, with $\mathcal{S}=\mathcal{T}$. For a typical (i.e., discrete) ping schedule, the

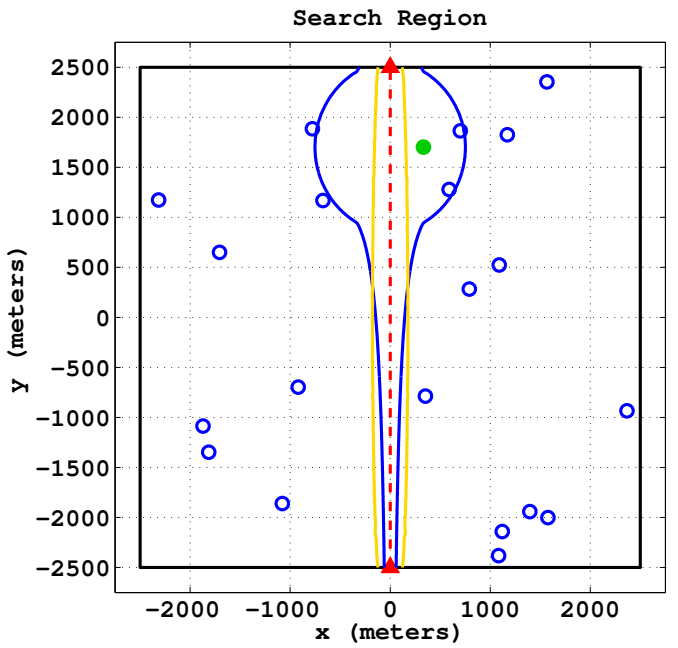

Fig. 2. Detection region $\Omega$ (solid blue lines) and average detection region $\bar{\Omega}$ (solid gold lines) about target track (dashed red line) for a multistatic sensor field with $N_{S}=1$ source (filled green circle). A sample of $N_{R}$ receivers from a uniform distribution are shown as unfilled blue circles.

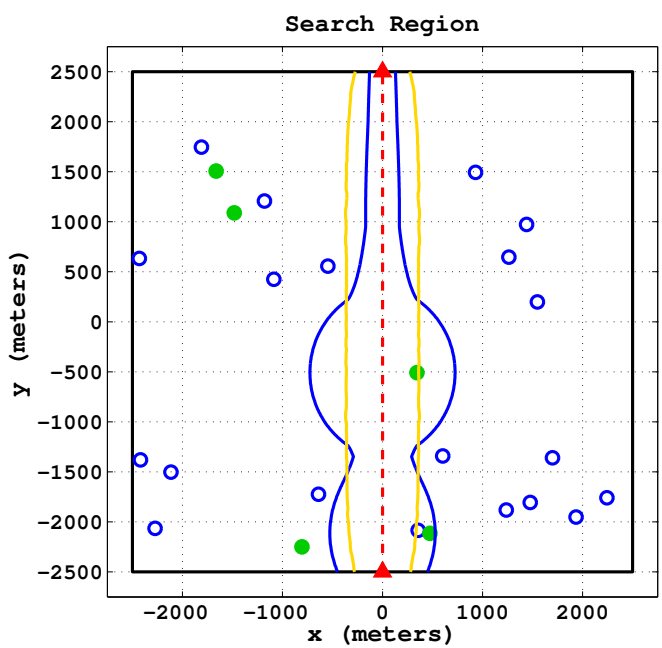

Fig. 3. Detection region $\Omega$ (solid blue lines) and average detection region $\bar{\Omega}$ (solid gold lines) about target track (dashed red line) for a multistatic sensor field with $N_{S}=5$ sources (filled green circles). A sample of $N_{R}$ receivers from a uniform distribution are shown as unfilled blue circles.

receiver detection region $\Omega$ may not be connected. However, the continuous model for $\mathcal{S}$ provides an upper bound on $\Omega$, and hence an upper bound on the search effectiveness of the sensor field.

The receiver detection regions shown in Figures 2 and 3 are for known source locations. If these locations are not known, then a reasonable alternative is to compute the expected receiver detection region $\bar{\Omega} \equiv \bigcup_{t \in \mathcal{S}} \bar{\Omega}_{t}$, with the region $\bar{\Omega}_{t}$ defined by

$$
\bar{\Omega}_{t} \equiv\left\{\vec{x} \in \mathcal{A}:\left\|\vec{x}-\vec{x}_{T}(t)\right\| \leq \bar{R}_{d}\left(\vec{x}_{T}(t)\right)\right\},
$$

where the average receiver detection range $\bar{R}_{d}\left(\vec{x}_{T}(t)\right)$ for a target at location $\vec{x}(t)$ is given by (15). The expected receiver detection region $\bar{\Omega}$ for $N_{S}=1$ and $N_{S}=5$ sources, uniformly 
(randomly) distributed in the search region $\mathcal{A}$, are plotted in Figures 2 and 3 , respectively. These regions are computed by sampling from the source location distribution $f$ at a sequence of points along the target track to estimate the density $f_{r_{(1)}(t)}$ at each point, and then using (15) to compute the average receiver detection range $\bar{R}_{d}$ at each of these points.

\section{Probability of Successful Search for Multistatic Network}

Our measure of the search effectiveness of multistatic sensor fields is the same as that for passive sensor fields, namely the probability of successful search $P_{S S}(k)$, defined as the probability of getting at least $k$ detections (from $k$ different receivers) during the search interval $\mathcal{T}$, and given by expressions (6), (7), and (8). The only difference between the calculation of $P_{S S}(k)$ for passive and multistatic fields is in the calculation of the probability $\phi$, given by (7), of finding a receiver in the detection region $\Omega$. Calculation of this probability involves integrating the receiver location density function $g$ over the set $\Omega$, and thus the difficulty of this calculation depends on the shape of this region. This shape can be quite complicated for multistatic sensor fields, as indicated by the examples in Figures 2 and 3.

If the source locations are known, corresponding to a source location density function $f$ with equal amounts of probability mass concentrated at each known source location, then the receiver detection region $\Omega$ is given precisely by the union of sets $\Omega_{t}$ as defined by (16). Examples of $\Omega$ in this case are the regions contained by the solid blue lines in Figures 2 and 3 . If the source locations are not known, then one approach is to calculate $P_{S S}(k)$ for the average detection region $\bar{\Omega}$ given by the union of sets $\bar{\Omega}_{t}$ as defined by (18). Examples of $\bar{\Omega}$ are the regions contained by the solid gold lines in Figures 2 and 3 . We denote this search effectiveness measure by $P_{S S}(k ; E[\Omega])$ to emphasize that averaging over the receiver detection region $\Omega$ is internal to the probability of successful search calculation in this case.

Another alternative for determining the search effectiveness of the field when the source locations are not known precisely is to compute the expected value of $P_{S S}(k)$ with respect to the random variables that describe the receiver detection region $\Omega$. We denote the search effectiveness measure in this case by $E\left[P_{S S}(k ; \Omega)\right]$ to emphasize that averaging over $\Omega$ is external to the probability of successful search calculation. In the remainder of this section, we propose an approximation to $E\left[P_{S S}(k ; \Omega)\right]$ that is straightforward to compute. In Section IV we compare this approximation the approximation $P_{S S}(k ; E[\Omega])$, and with values of $P_{S S}(k)$ obtained through Monte Carlo simulation.

Observe that the receiver detection range $R_{d}$ given by (12) is bounded from above by $r_{0}^{2} / r_{(1)}^{*}$, where $r_{i}^{*}$ denotes the range from the target to the $i$ th source at closest-point-of-approach $(\mathrm{CPA})$, and $r_{(1)}^{*}$ denotes the CPA range for the source with the smallest CPA range. Indeed,

$$
\begin{aligned}
\frac{r_{0}^{2}}{r_{S_{(1)}}(t)} & \leq \frac{r_{0}^{2}}{\min _{t} r_{S_{(1)}}(t)}=\frac{r_{0}^{2}}{\min _{t} \min _{i} r_{S_{i}}(t)} \\
& =\frac{r_{0}^{2}}{\min _{i} \min _{t} r_{S_{i}}(t)}=\frac{r_{0}^{2}}{\min _{i} r_{i}^{*}}=\frac{r_{0}^{2}}{r_{(1)}^{*}} .
\end{aligned}
$$

Let $\Omega_{t}^{*} \equiv \Omega_{t}^{*}\left(R_{d}^{*}\right)$ denote the detection region with radius $R_{d}^{*}=r_{0}^{2} / r_{(1)}^{*}$ about the target at location $\vec{x}_{T}(t)$, that is,

$$
\Omega_{t}^{*}\left(R_{d}^{*}\right)=\left\{\vec{x} \in \mathcal{A}:\left\|\vec{x}-\vec{x}_{T}(t)\right\| \leq R_{d}^{*}\right\},
$$

and let $\Omega^{*} \equiv \Omega^{*}\left(R_{d}^{*}\right)=\bigcup_{t \in \mathcal{S}} \Omega_{t}^{*}\left(R_{d}^{*}\right)$. Then $\Omega \subset \Omega^{*}$, and the probability of successful search $P_{S S}\left(k ; \Omega^{*}\right)$ associated with the receiver detection region $\Omega^{*}$ is an upper bound for the probability of successful search $P_{S S}(k ; \Omega)$ associated with the region $\Omega$. Moreover, given the density function $f_{r_{(1)}^{*}}$ for the random variable $r_{(1)}^{*}$, the density function $f_{R_{d}^{*}}$ for the upper bound detection range $R_{d}^{*}$ can be obtained, and the expected value of $P_{S S}\left(k ; \Omega^{*}\right)$ can be computed via the integral

$$
E\left[P_{S S}\left(k ; \Omega^{*}\right)\right]=\int P_{S S}\left(k ; \Omega^{*}(R)\right) f_{R_{d}^{*}}(R) d R .
$$

\section{ExAmPle: Uniform Source ANd Receiver DisTRIBUTIONS}

Consider the example of Figures 2 and 3, where the search region $\mathcal{A}$ is a $2 L \times 2 L$ box centered at the origin, and the sources and receivers are identically and independently distributed uniformly (randomly) over $\mathcal{A}$. The target track for this scenario begins at location $\vec{x}_{T}\left(t_{0}\right)=(0,-L)$ and ends at location $\vec{x}_{T}\left(t_{0}+\Delta t\right)=(0, L)$. Evidently, the CPA range $r_{i}^{*}$ from the $i$ th source at location $\vec{x}_{S_{i}}=\left(x_{S_{i}}, y_{S_{i}}\right)$ to the target track is equal to $\left|x_{S_{i}}\right|$, and since $x_{S_{i}}$ is distributed uniformly in the interval $[-L, L]$, it follows that $r_{i}^{*}$ is distributed uniformly in the the interval $[0, L]$. Thus, the density function $f_{r_{i}^{*}}$ for the CPA range from the $i$ th source to the target track is given by

$$
f_{r_{i}^{*}}(\rho)= \begin{cases}\frac{1}{L}, & 0 \leq \rho \leq L, \\ 0, & \text { otherwise }\end{cases}
$$

Given this density function, the density function $f_{r_{(1)}^{*}}$ for the smallest CPA range $r_{(1)}^{*}$ is obtained using well known results from the theory of order statistics, specifically [11, Theorem 5.5.2]:

$$
f_{r_{(1)}^{*}}(\rho)= \begin{cases}\frac{N_{S}}{L}\left(\frac{L-\rho}{L}\right)^{N_{S}-1}, & 0 \leq \rho \leq L, \\ 0, & \text { otherwise. }\end{cases}
$$

Finally, the density function for the upper bound detection range $R_{d}^{*}=r_{0}^{2} / r_{(1)}^{*}$ is obtained from (23) using standard procedures for transformations of random variables. The result is

$$
f_{R_{d}^{*}}(R)= \begin{cases}\frac{N_{S}}{L-r_{0}^{2} / L}\left(\frac{L-r_{0}^{2} / R}{L-r_{0}^{2} / L}\right)^{N_{S}-1} \frac{r_{0}^{2}}{R^{2}}, & \frac{r_{0}^{2}}{L} \leq R \leq L, \\ 0, & \text { otherwise. }\end{cases}
$$


For this scenario, the receiver detection region $\Omega^{*}(R)$ for a given detection range $R$ is just the locus of points in $\mathcal{A}$ with $x$-coordinates within $R$ of the target track. It follows that the probability $\phi$ of finding a receiver in $\Omega^{*}(R)$ is just $\phi=$ $R / L$. Substituting this result into (6) gives the sensor-level probability of successful search $p$ in terms of the detection range $R$ :

$$
p=1-\exp \left(-P_{d} R / L\right) .
$$

Expressions (25) and (8) then combine to give the field-level probability of successful search as a function of the detection range $R$. This result, combined with the upper bound detection range density function (24), can be used in (21) to compute the average value of probability of successful search for the upper bound receiver detection region $\Omega^{*}$.

The expected probability of successful search $E\left[P_{S S}\left(k ; \Omega^{*}\right)\right]$ for the scenario described in this section is plotted (with gray dashed lines) in Figures 4 through 6 as a function of the number of the number of receivers $N_{R}$ for various values of number of sources $N_{S}$, monostatic detection range $r_{0}$, and required detections $k$. The search area for this example is a 5 kilometer by 5 kilometer box. For comparison, the probability of successful search $P_{S S}(k ; E[\Omega])$ based on the expected receiver detection region $\bar{\Omega}=E[\Omega]$ is also plotted (with orange dotted lines) in these figures, as well as the the probability of successful search computed via Monte Carlo simulation (plotted with solid purple lines). For each of the simulations and each value of $N_{R}$ (from 5 to 50 receivers), we sampled 10,000 sets of $N_{S}$ sources and $N_{R}$ receivers uniformly (randomly) distributed over the search region $\mathcal{A}$, and counted the number of trials for which at least $k$ distinct receivers detected the target (at least once) during the search interval $\mathcal{T}$ for a specified value of $k$.

The plots in Figure 4 are for $k=1$ required detection and a monostatic detection range $r_{0}=500$ meters, and show how probability of successful search varies as a function of the number of sources $N_{S}$ (for 1,5 , and 10 sources). Not surprisingly, this probability increases with the numbers of sources and receivers. In all cases, the two approximations for $P_{S S}(k)$ show good agreement with the curve obtained from Monte Carlo simulation. In particular, the curve for the expected probability of successful search $E\left[P_{S S}\left(k ; \Omega^{*}\right)\right]$ bounds the simulation curve from above in all cases shown. We note, however, that this is not guaranteed; indeed, while it is true that $P_{S S}\left(k ; \Omega^{*}\right)$ is an upper bound for $P_{S S}(k)$ in all cases, this does does not imply $P_{S S}(k) \leq E\left[P_{S S}\left(k ; \Omega^{*}\right)\right]$.

Figure 5 show similar plots, but for $k=3$ required detections. The two approximations for $P_{S S}(k)$ are not as close to the values for $P_{S S}(k)$ obtained from simulation in this case, particularly with respect to the approximation $P_{S S}(k ; E[\Omega])$. Nevertheless, all three curves exhibit similar trends with increasing numbers of sources and receivers, and the expected probability of successful search $E\left[P_{S S}\left(k ; \Omega^{*}\right)\right]$ curves bound the simulation curves from above in almost all cases, except for those with small numbers of receivers (for example, in the case $N_{R}=5$ and $N_{S}=10$ ).
The final set of plots, in Figure 6, are for $k=3$ required detections and $N_{S}=5$ sources, and show how probability of successful search varies as a function of the monostatic detection range $r_{0}$ (for 300, 500, and 700 meters). The agreement between our two approximations for probability of successful search and the values of $P_{S S}(k)$ obtained via simulation clearly improves with increasing monostatic detection range. Again, in almost all cases the expected probability of successful search $E\left[P_{S S}\left(k ; \Omega^{*}\right)\right]$ curves bound the simulation curves from above.

\section{Summary, Conclusions, And Future Work}

We have developed an approximate performance model for undersea surveillance using multistatic sensor fields. The model provides an upper bound on the field-level detection performance that is achievable by systems comprised of separate sources and receivers, even in scenarios when multiple source/receiver detections are required for field-level detection decisions. The model's effectiveness and accuracy were demonstrated against simulated examples of varying numbers of sources and receivers in a homogeneous environment. These simulation comparisons showed good agreement between the model predictions and computationally cumbersome Monte Carlo simulations.

This performance model for multistatic sensor fields was developed as an extension of an existing performance model for distributed passive sensor networks that relies on a spatial density description of the sensor locations. One major benefit of these density-based approaches is their computational efficiency when compared to conventional Monte Carlo type, simulation-based approaches to field performance modeling. Furthermore, by using this density-based approach, we expect to readily adapt previous work in cost-effective passive sensor field sizing [1] to the sizing of multistatic fields. Other areas of current investigation include the use of these models in optimal positioning of sensor nodes for field control, as well as their use in providing computationally efficient methods to adapt a field to environmental changes.

\section{ACKNOWLEDGMENT}

This work was supported by the Office of Naval Research Code 321MS.

\section{REFERENCES}

[1] C. M. Traweek and T. A. Wettergren, "Efficient sensor characteristic selection for cost-effective distributed sensor networks," IEEE Journal of Oceanic Engineering, vol. 31, no. 2, pp. 480-486, April 2006.

[2] T. A. Wettergren, "Performance of search via track-before-detect for distributed sensor networks," IEEE Transactions on Aerospace and Electronic Systems, vol. 44, no. 1, pp. 314-325, January 2008.

[3] J. I. Bowen and R. W. Mitnick, "A multistatic performance prediction methodology," Johns Hopkins APL Technical Digest, vol. 20, no. 3, pp. 424-431, 1999.

[4] P. N. Ngatchou, W. L. J. Fox, and M. A. El-Sharkawi, "Multiobjective multistatic sonar sensor placement," in Proceedings of the 2006 IEEE Congress on Evolutionary Computations, Vancouver, BC, Canada, 1621 July 2006, pp. 2713-2719.

[5] R. J. Urick, Principles of Underwater Sound, 3rd ed. New York: McGraw-Hill, 1983. 
[6] A. Papoulis, Probability, Random Variables, and Stochastic Processes, 3rd ed. New York: McGraw-Hill, 1991.

[7] H. Cox, "Fundamentals of bistatic active sonar," BBN Systems and Technologies Corporation, Arlington, Virginia, Technical Memorandum No. W1068, July 1988.

[8] N. J. Willis, Bistatic Radar, 2nd ed. SciTech Publishing, 2005.

[9] A. E. Hirst and E. K. Lloyd, "Cassini, his ovals, and a space probe to Saturn," The Mathematical Gazette, vol. 81, no. 492, pp. 409-421, November 1997.

[10] D. W. Krout, M. A. El-Sharkawi, W. L. J. Fox, and M. U. Hazen, "Intelligent ping sequencing for multistatic sonar systems," in Proceedings of the 9th International Conference on Information Fusion, 10-13 July 2006, pp. 1-6.

[11] G. Casella and R. L. Berger, Statistical Inference. Belmont, California: Duxbury Press, 1990.

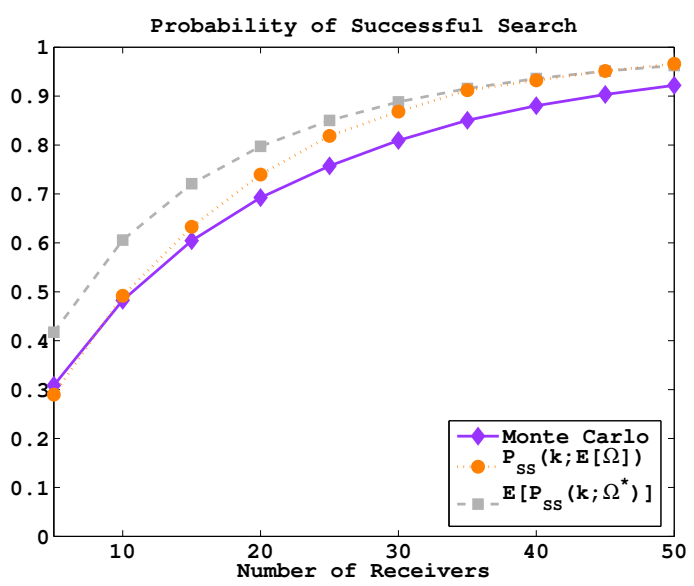

(a) $k=1, N_{S}=1$.

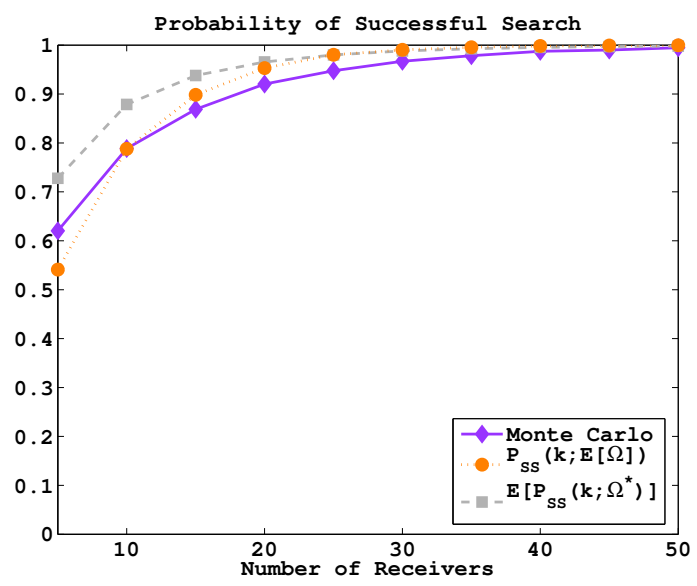

(b) $k=1, N_{S}=5$.

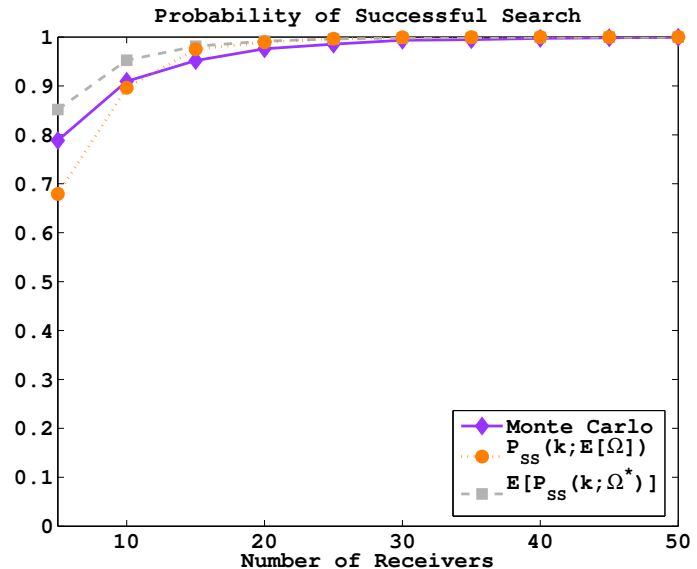

(c) $k=1, N_{S}=10$.

Fig. 4. Search effectiveness, for $k=1$ required detections, of multistatic sensor field with various numbers of sources $N_{S}$ and a monostatic detection range $r_{0}=500 \mathrm{~m}$ 


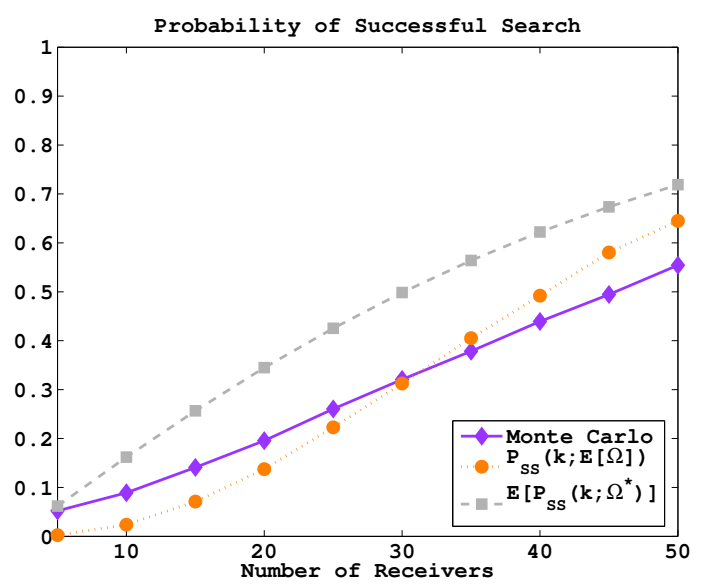

(a) $k=3, N_{S}=1$.

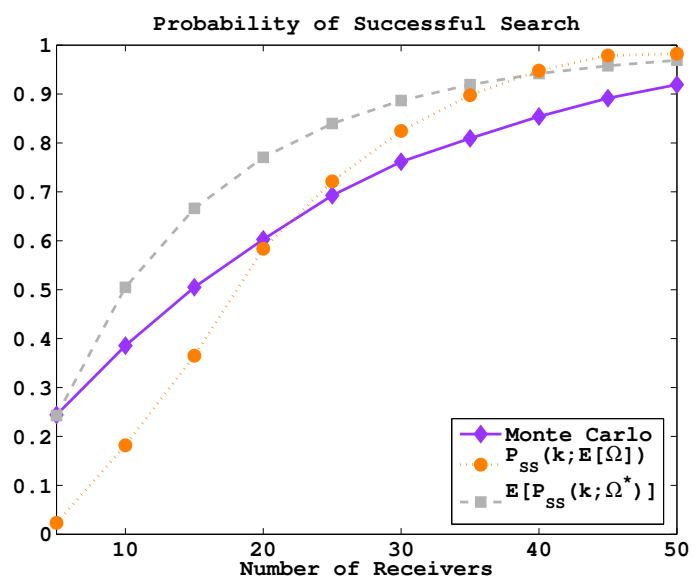

(b) $k=3, N_{S}=5$.

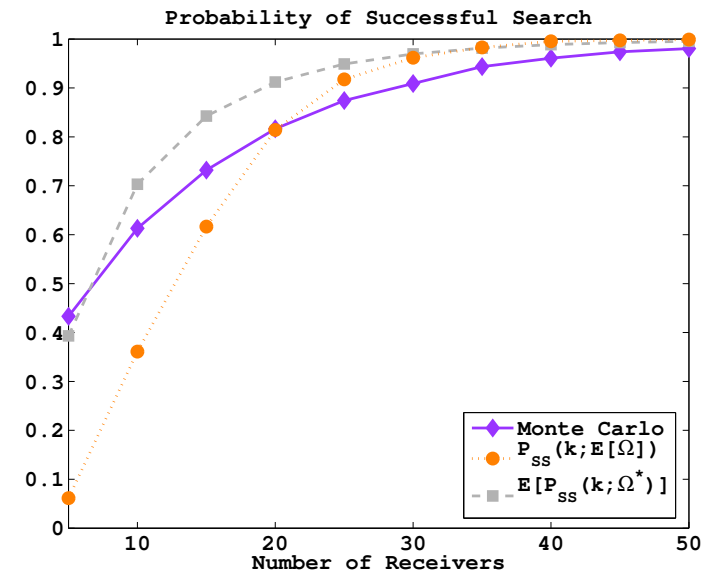

(c) $k=3, N_{S}=10$.

Fig. 5. Search effectiveness, for $k=3$ required detections, of multistatic sensor field with various numbers of sources $N_{S}$ and a monostatic detection range $r_{0}=500 \mathrm{~m}$.

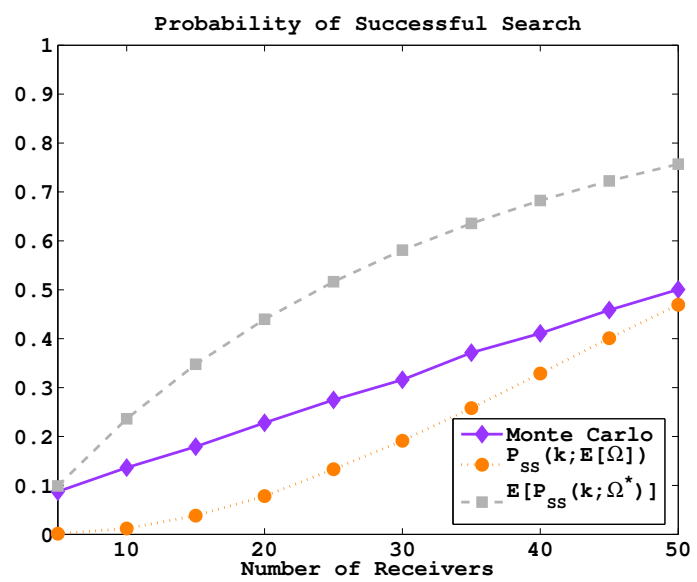

(a) $k=3, r_{0}=300 \mathrm{~m}$.

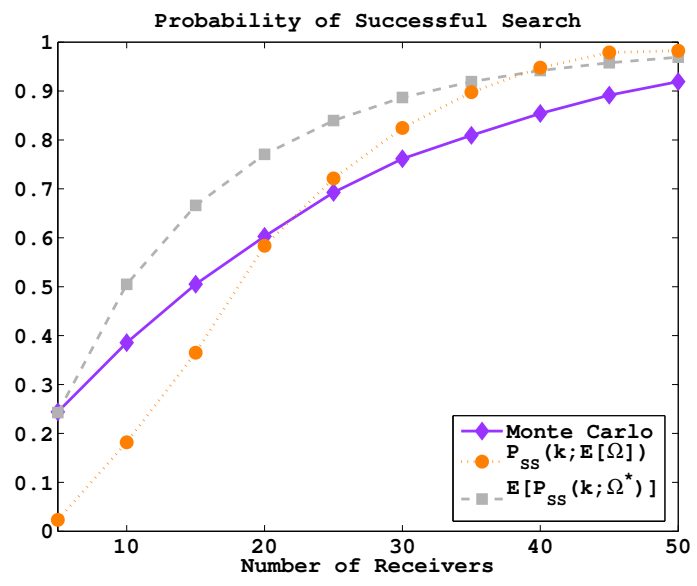

(b) $k=3, r_{0}=500 \mathrm{~m}$.

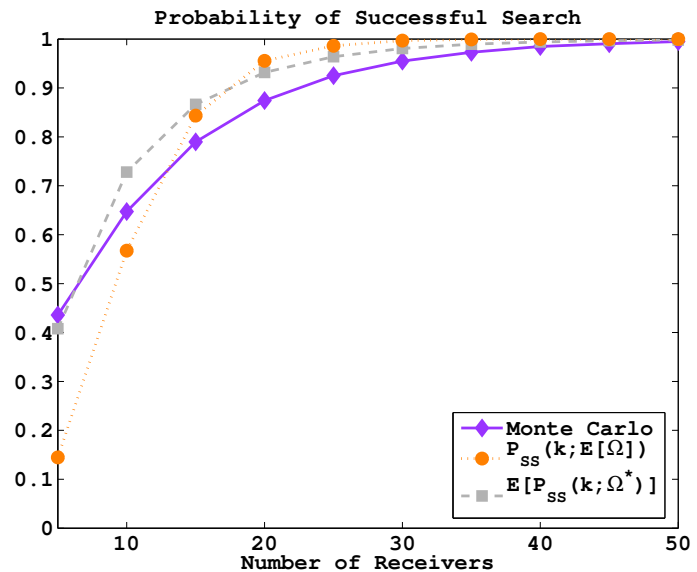

(c) $k=3, r_{0}=700 \mathrm{~m}$.

Fig. 6. Search effectiveness, for $k=3$ required detections, of multistatic sensor field $N_{S}=5$ sources and various values for monostatic detection range $r_{0}$. 